\title{
Diagnosing Surface Mixed Layer Dynamics from High-Resolution Satellite Observations: Numerical Insights
}

\author{
Aurelien L. Ponte And PATrice Klein \\ Laboratoire de Physique des Océans, IFREMER-CNRS-IRD-UBO, Plouzané, France \\ XAVIER CAPET \\ LOCEAN, IPSL, Paris, France \\ PiERre-Yves LE TrAON AND BERTRAND CHAPRON \\ Laboratoire d'Océanographie Spatiale, IFREMER, Plouzané, France \\ PASCALE LHERMINIER \\ Laboratoire de Physique des Océans, IFREMER-CNRS-IRD-UBO, Plouzané, France
}

(Manuscript received 27 July 2012, in final form 1 March 2013)

\begin{abstract}
High-resolution numerical experiments of ocean mesoscale eddy turbulence show that the wind-driven mixed layer (ML) dynamics affects mesoscale motions in the surface layers at scales lower than $O(60 \mathrm{~km})$. At these scales, surface horizontal currents are still coherent to, but weaker than, those derived from sea surface height using geostrophy. Vertical motions, on the other hand, are stronger than those diagnosed using the adiabatic quasigeotrophic (QG) framework. An analytical model, based on a scaling analysis and on simple dynamical arguments, provides a physical understanding and leads to a parameterization of these features in terms of vertical mixing. These results are valid when the wind-driven velocity scale is much smaller than that associated with eddies and the Ekman number (related to the ratio between the Ekman and ML depth) is not small. This suggests that, in these specific situations, three-dimensional ML motions (including the vertical velocity) can be diagnosed from high-resolution satellite observations combined with a climatological knowledge of ML conditions and interior stratification.
\end{abstract}

\section{Introduction}

Conventional altimetric observations that capture sea surface height (SSH) structures only down to mesoscale, $O(100 \mathrm{~km})$, provide estimates of surface currents at these scales using geostrophy (Dohan and Maximenko 2010). Recent studies however stirred interest for smaller scales down to $O(10 \mathrm{~km})$ because of their contribution to vertical transports and impact on large-scale ocean properties via nonlinear interactions (Capet et al. 2008b; Klein et al. 2008; Lévy et al. 2010). Within this context future altimetric missions, such as the Surface Water and

Corresponding author address: Patrice Klein, Laboratoire de Physique des Océans, Ifremer-CNRS-UBO-IRD, 29280 Plouzané, France.

E-mail: patrice.klein@ifremer.fr
Ocean Topography (SWOT) mission, which aims at a tenfold improvement of spatial resolution ( $\mathrm{Fu}$ and Ferrari 2008), combined with high-resolution satellite imagery (Kudryavtsev et al. 2012), offer promising perspectives.

Numerical studies have examined the potential of high-resolution SSH to diagnose such small-scale motions, including the vertical velocity field (Isern-Fontanet et al. 2006, 2008; Klein et al. 2009). The methods employed rely on adiabatic quasigeostrophy (QG) [and more precisely on surface QG (SQG), see Lapeyre and Klein (2006)], which only requires knowledge of the ocean interior large-scale potential vorticity (PV). These methods appear to successfully capture three-dimensional motions down to $O(10 \mathrm{~km})$ within the first $500 \mathrm{~m}$ below the surface mixed layer (ML). They, however, fail to retrieve ML motions (Isern-Fontanet et al. 2008; Klein et al. 2009), which motivates the present work. 
We address this issue with high-resolution simulations of mesoscale turbulence (described in section 2a). Departures of ML motions from adiabatic QG are observed in the simulations only for scales smaller than $60 \mathrm{~km}$, with opposite consequences on horizontal and vertical flow variances (section $2 b$ ). The analytical development, in section 3, that relies on a scaling analysis and on simple dynamical arguments, provides a physical understanding and a parameterization for these departures in terms of vertical mixing and ML depth. The results suggest that, in situations where the surface eddy velocity scale is much larger than the wind-driven velocity and for $O(1)$ Ekman number [i.e., an $O(1)$ ratio between the Ekman depth and the depth scale of geostrophic motions affected by ML dynamics, i.e., the ML depth], ML motions (including the vertical velocity) can be diagnosed using high-resolution satellite observations provided some knowledge of large-scale ML characteristics (section 4) and interior stratification.

\section{Numerical results}

\section{a. Simulations}

Two high-resolution $(2 \mathrm{~km}$ in the horizontal and 100 vertical levels) simulations of forced oceanic mesoscale turbulence (in a $1000 \mathrm{~km} \times 2000 \mathrm{~km}$ domain with a 4000-m depth) are analyzed. The mesoscale eddy field is produced by the instability of a large-scale baroclinic westerly flow in a zonal $\beta$-plane channel centered at $45^{\circ} \mathrm{N}$ (corresponding to a Coriolis frequency $f=10^{-4} \mathrm{~s}^{-1}$ ). The total eddy kinetic energy is in statistical equilibrium after a spinup of 400 days corresponding to a root-mean-square (rms) value of the surface eddy velocity: $u_{\text {Eddy }}=0.2 \mathrm{~m} \mathrm{~s}^{-1}$ [see Klein et al. (2008) for a description of the characteristics of the turbulent eddy field]. The first simulation (described in Klein et al. 2009) includes an active mixed layer with a depth $H=65 \mathrm{~m}$, whose characteristics are fully described in Danioux et al. (2011). The mixed layer is forced by uniform air-sea fluxes that include a wind stress (Fig. 1) using a realistic wind time series. This time series comes from meteorological data sampled every $3 \mathrm{~h}$ on the weather ship KILO located in the North Atlantic [see Klein et al. (2004) for a description of these data]. The wind direction and amplitude significantly vary in time (Fig. 1). Using an rms value of the windstress, $\tau_{\text {rms }} \approx$ $10^{-4} \mathrm{~m}^{2} \mathrm{~s}^{-2}$, leads to a mean wind-driven velocity $u_{w}=$ $\tau_{\text {rms }} / f H \approx 1.510^{-2} \mathrm{~m} \mathrm{~s}^{-1}$ and a ratio $u_{\text {Eddy }} / u_{w} \approx 13$. The mean turbulent vertical viscosity within the ML is $A_{v} \approx 4$ $\times 10^{-2} \mathrm{~m}^{2} \mathrm{~s}^{-1}$ leading to an Ekman depth, $\delta_{e}=\left(2 A_{v} / f\right)^{1 /}$ $2 \approx 30 \mathrm{~m}$. Model outputs are low-pass filtered to eliminate near-inertial motions. The second simulation has no airsea flux and, thus, no ML (as in Klein et al. 2008) and is used to contrast physical processes present with and without a mixed layer.

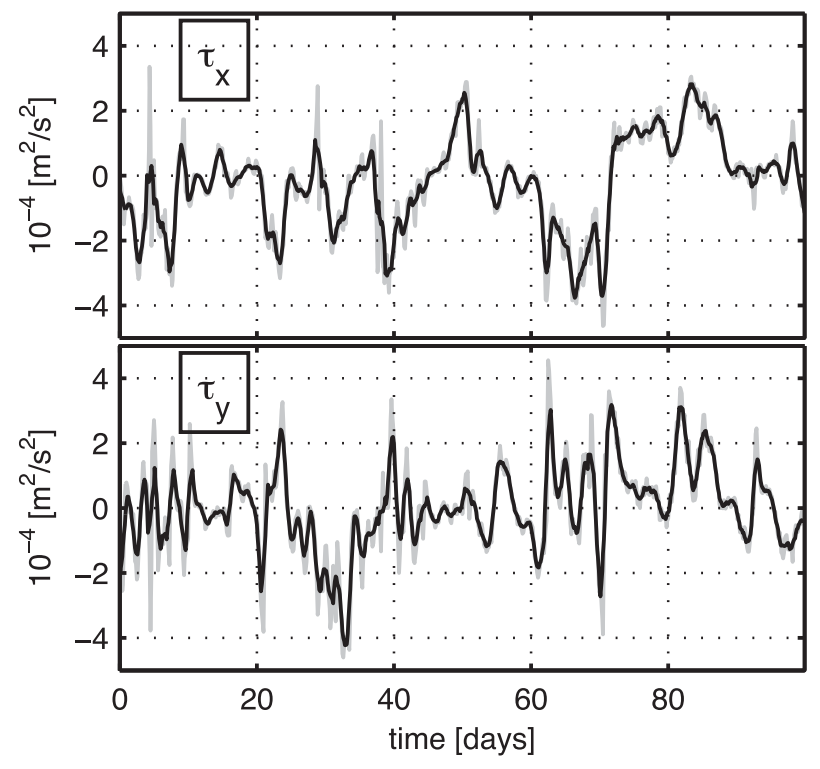

FIG. 1. Time series of three-hourly (gray curves) $x$ and $y$ components of the wind stress used in the ML simulation; time-averaged values of $x$ and $y$ wind stresses are $-2 \times 10^{-5}$ and $-6 \times$ $10^{-7} \mathrm{~m}^{2} \mathrm{~s}^{-2}$, respectively. The wind stress rms value $\tau_{\mathrm{rms}}$ is close to $10^{-4} \mathrm{~m}^{2} \mathrm{~s}^{-2}$. The black curves are the time series averaged over $24 \mathrm{~h}$.

The relative vorticity $\zeta$ and density $\rho$ fields at $40 \mathrm{~m}$ (Figs. $2 \mathrm{a}-\mathrm{d})$ reveal energetic mesoscale eddies and submesoscale structures in both simulations. The Rossby number (estimated as $\left.\mathrm{Ro} \approx \zeta_{\mathrm{rms}} / f\right)$ is about $0.2(0.6)$ for the simulation with (without) an ML. The density field exhibits strong density fronts aligned with relative vorticity gradients. Vertical motions at a 40-m depth $w_{40}$ with and without an ML (respectively Figs. 2f,e) have a magnitude up to $50 \mathrm{~m} \mathrm{~d}^{-1}$ and are closely associated with density fronts (Figs. 2b,a). Figure $2 \mathrm{f}$ further indicates that spatial patterns of vertical motions with mixed layer (that exhibit filamentary structures astride density fronts) much differ from those without an ML (Fig. 2e) that display alongfront alternating signs. A detailed analysis (not shown) indicates that ML vertical motions are actually correlated with the Laplacian of the density (see section 3).

Although the Rossby number is large, one specific property of the mesoscale and submesoscale eddy field in the simulation without an ML is that it is almost geostrophically balanced (as noted in Klein et al. 2008). One explanation (detailed in Klein et al. 2008) is that the dynamics in the upper layers is dominated by the SQG dynamics. On the other hand, the relative vorticity has been found to be strongly skewed (displaying positive values up to $3 f$ but negative values not smaller than $-f)$. This means that ageostrophic relative vorticity can be significant, but this only concerns scales smaller than $10 \mathrm{~km}$ (Klein et al. 2008, see their Fig. 6). The same properties have been reported in Capet et al. (2008a) 

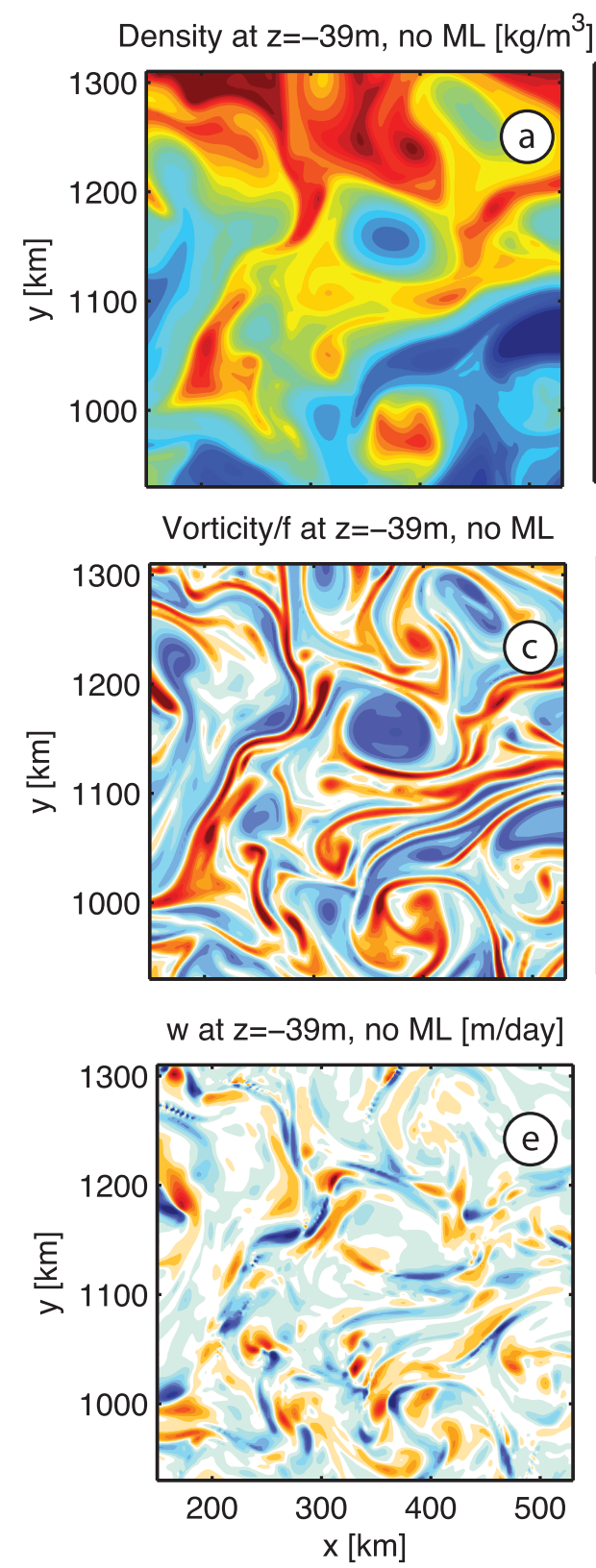
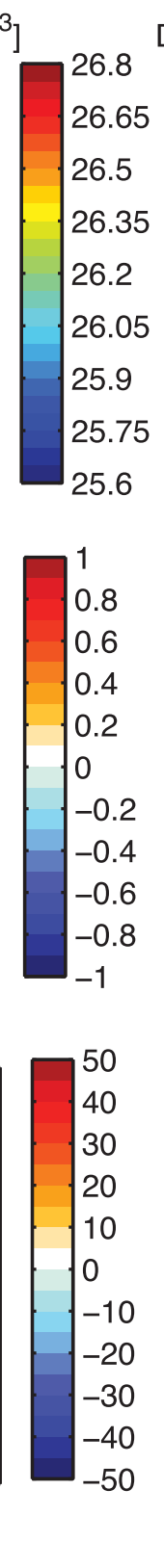

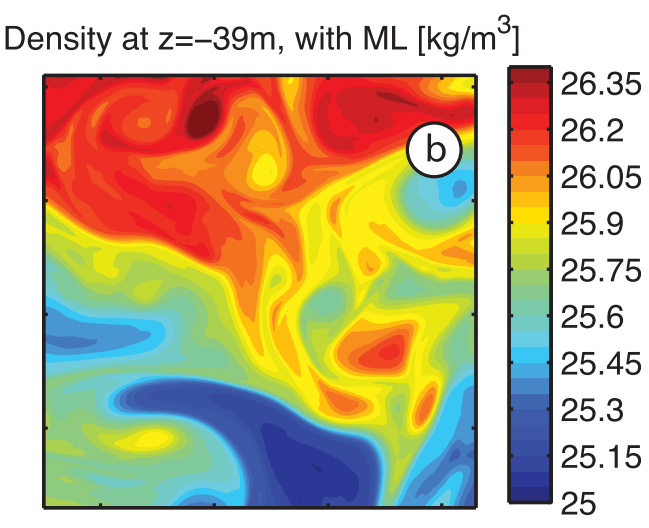

Vorticity/f at $z=-39 \mathrm{~m}$, with $\mathrm{ML}$

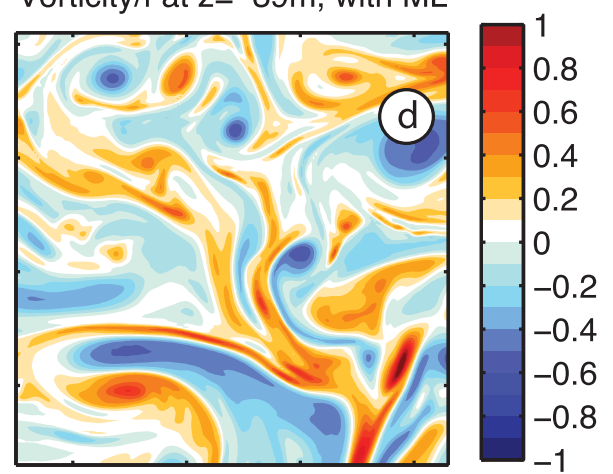

w at $z=-39 m$, with $M L[m / d a y]$

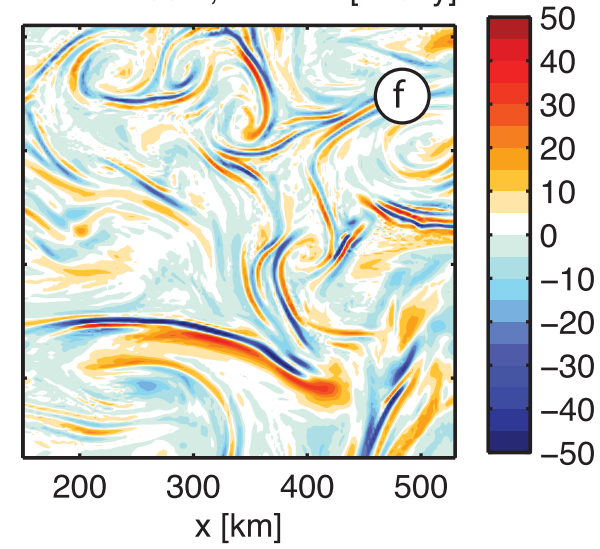

FIG. 2. Snapshots of (a),(b) the density field, (c),(d) the relative vorticity field normalized by $f$, and (e),(f) the vertical velocity field at $40 \mathrm{~m}$ (left) without and (right) with ML: Vertical velocity $\left(\mathrm{m} \mathrm{d}^{-1}\right)$.

who analyzed a numerical simulation with a different configuration.

\section{b. Horizontal and vertical motions in the upper ocean}

Model motions are first compared with those diagnosed from adiabatic QG. Within this framework surface horizontal motions observed in the simulation $\mathbf{u}_{s}$, are compared with the geostrophic motions $\mathbf{u}_{g}$ estimated as $\mathbf{u}_{g}=g f^{-1} \mathbf{k} \times \nabla \eta$ (with $\eta$ the sea surface height, $g$ the gravity constant, and $\mathbf{k}$ the unit vertical vector). Vertical motions at $40 \mathrm{~m} w_{40}$, are compared with those diagnosed from the Omega equation (Hoskins et al. 1978; Pollard and Regier 1992; Rudnick 1996; Giordani and Caniaux 2005; Nagai et al. 2006) forced by the kinematic deformation component of the $\mathbf{Q}$ vector

$$
N^{2} \Delta w_{\mathrm{QG}}+f^{2^{2}} \frac{\partial^{2} w_{\mathrm{QG}}}{\partial z^{2}}=\nabla \cdot \mathbf{Q}_{\mathbf{k d}},
$$


no ML
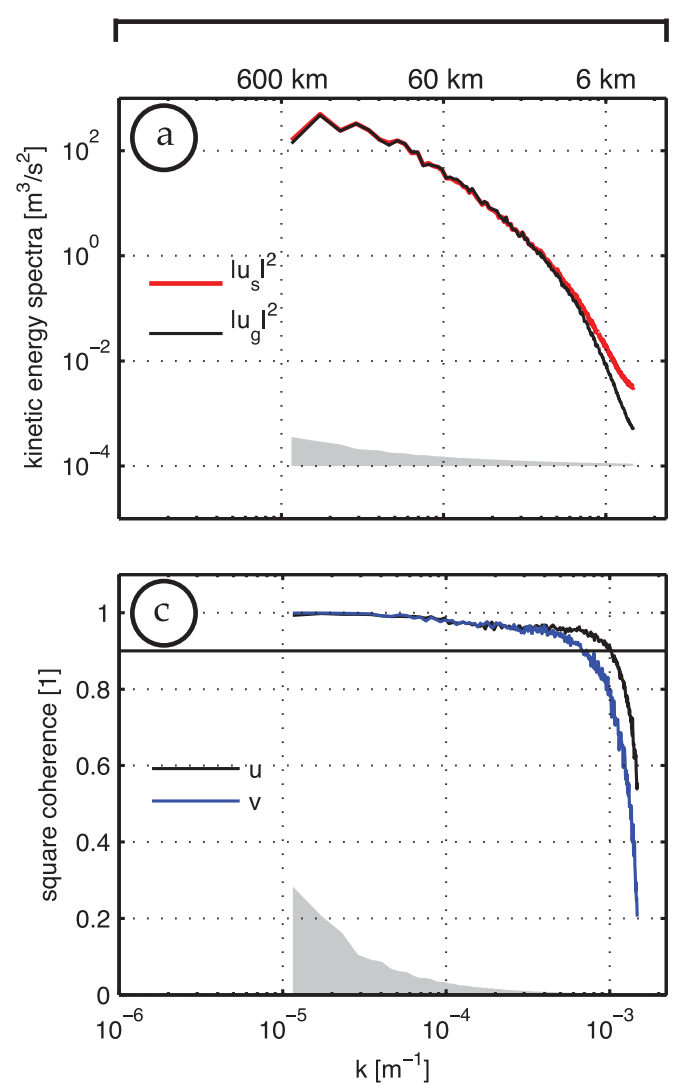

with ML
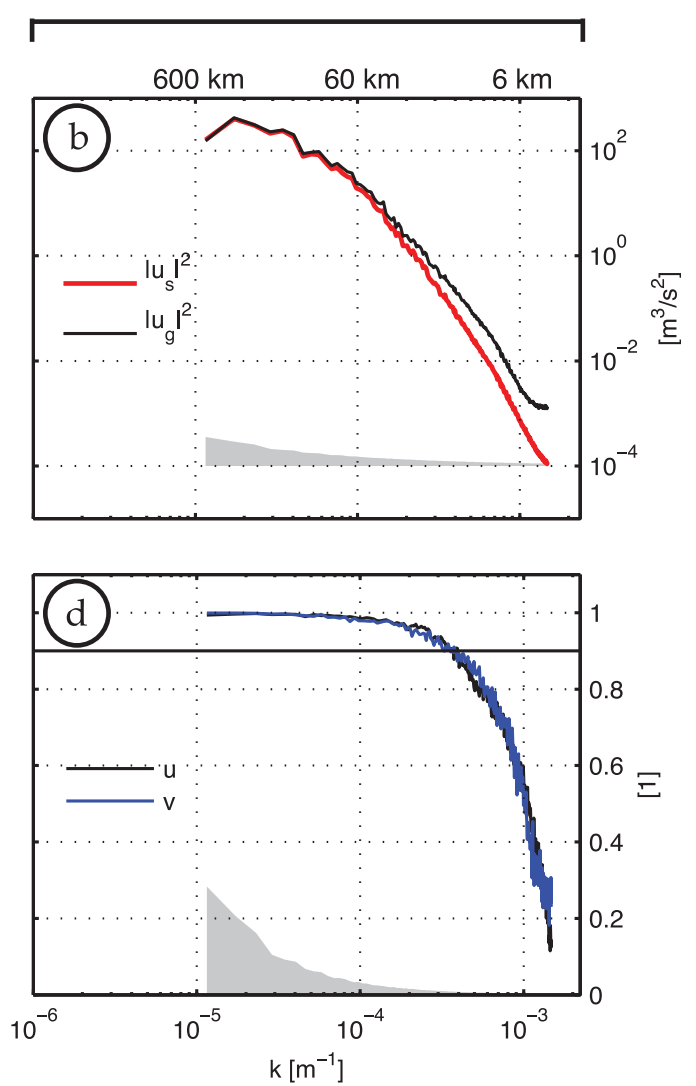

FIG. 3. Surface velocity spectrum estimated from the SSH using geostrophy (black curve), from the surface velocity (red curve) (a) without and (b) with ML. Units on the vertical axis are in $\mathrm{m}^{3} \mathrm{~s}^{-2}$. On the horizontal axis, $k=10^{-4} \mathrm{rad} \mathrm{m}^{-1}$ corresponds to a wavelength of $60 \mathrm{~km}$. (c),(d) Spectral squared coherence between the geostrophic velocities and the surface velocities without (c) and with ML (d). Gray shadings represent $95 \%$ confidence intervals. Spectra are $2 \mathrm{D}$ and summed over $\left(k_{x}, k_{y}\right)$ with equal total wavenumbers $k=\sqrt{k_{x}^{2}+k_{y}^{2}}$.

with $\mathbf{Q}_{\mathbf{k d}}=\left(\nabla \mathbf{u}_{h}\right)^{t} \cdot \nabla \rho ; N$ is the Brunt-Väisälä frequency, $\mathbf{u}_{h}$ the nondivergent part of horizontal motions, and $\nabla$ the horizontal gradient operator. Departures of near-surface 3D motions from adiabatic QG are best understood in horizontal spectral space.

With a mixed layer, the spectrum of surface horizontal motions $\mathbf{u}_{s}$ (red curve on Fig. $3 \mathrm{~b}$ ) is comparable to the $\mathbf{u}_{g}$ spectrum (black curve on Fig. 3b) for scales between 60 and $600 \mathrm{~km}$ with a slope close to $K^{-2}$, with $K$ the wavenumber modulus. For smaller scales, however, the $\mathbf{u}_{s}$ spectrum is steeper than the $\boldsymbol{u}_{g}$ spectrum, which indicates a departure from geostrophy. In physical space, the differences between $\left|\boldsymbol{u}_{s}\right|$ and $\left|\boldsymbol{u}_{g}\right|$ (not shown) mostly appear in thin structures. The $\boldsymbol{u}_{s}$ energy level is about $20 \%$ smaller than that of the $\boldsymbol{u}_{g}$ energy level for scales smaller than $100 \mathrm{~km}$. At last the spectral coherence between $\boldsymbol{u}_{s}$ and $\boldsymbol{u}_{g}$ (Fig. 3d) remains remarkably close to one down to scales of about $15 \mathrm{~km}$. Without ML on the other hand, $\boldsymbol{u}_{s}$ and $\boldsymbol{u}_{g}$ spectra (Fig. 3a) are almost identical [with a $K^{-2}$ slope over a large spectral range as already reported in Klein et al. (2008)]. The spectral coherence between $\boldsymbol{u}_{s}$ and $\boldsymbol{u}_{g}$ is slightly better without ML and remains close to one down to wavelengths of $8 \mathrm{~km}$ (Fig. 3c). Surface currents without ML are therefore almost in geostrophic equilibrium even in the small-scale range, contrary to what is observed in the simulation with an ML.

The spectrum of vertical velocity at $40 \mathrm{~m}$ (i.e., $w_{40}$ ) in the simulation with ML (black curve on Fig. 4b) overlaps the spectrum of the vertical velocity (i.e., $w_{\mathrm{QG}}$ ) diagnosed from (1) (blue curve on Fig. 4b) for scales larger than $60 \mathrm{~km}$. For smaller scales, the $w_{40}$ spectrum is more energetic than the $w_{\mathrm{QG}}$ spectrum indicating a departure from QG. This departure corresponds to a 4 times increase of variance and is opposite to what occurs for horizontal motions (displayed by Fig. 3b). The spectral coherence between $w_{40}$ and $w_{\mathrm{QG}}$ is above 0.6 only for scales greater than $60 \mathrm{~km}$ (Fig. 4d). Without the ML, the $w_{\mathrm{QG}}$ spectrum is closer over the entire spectral range to 

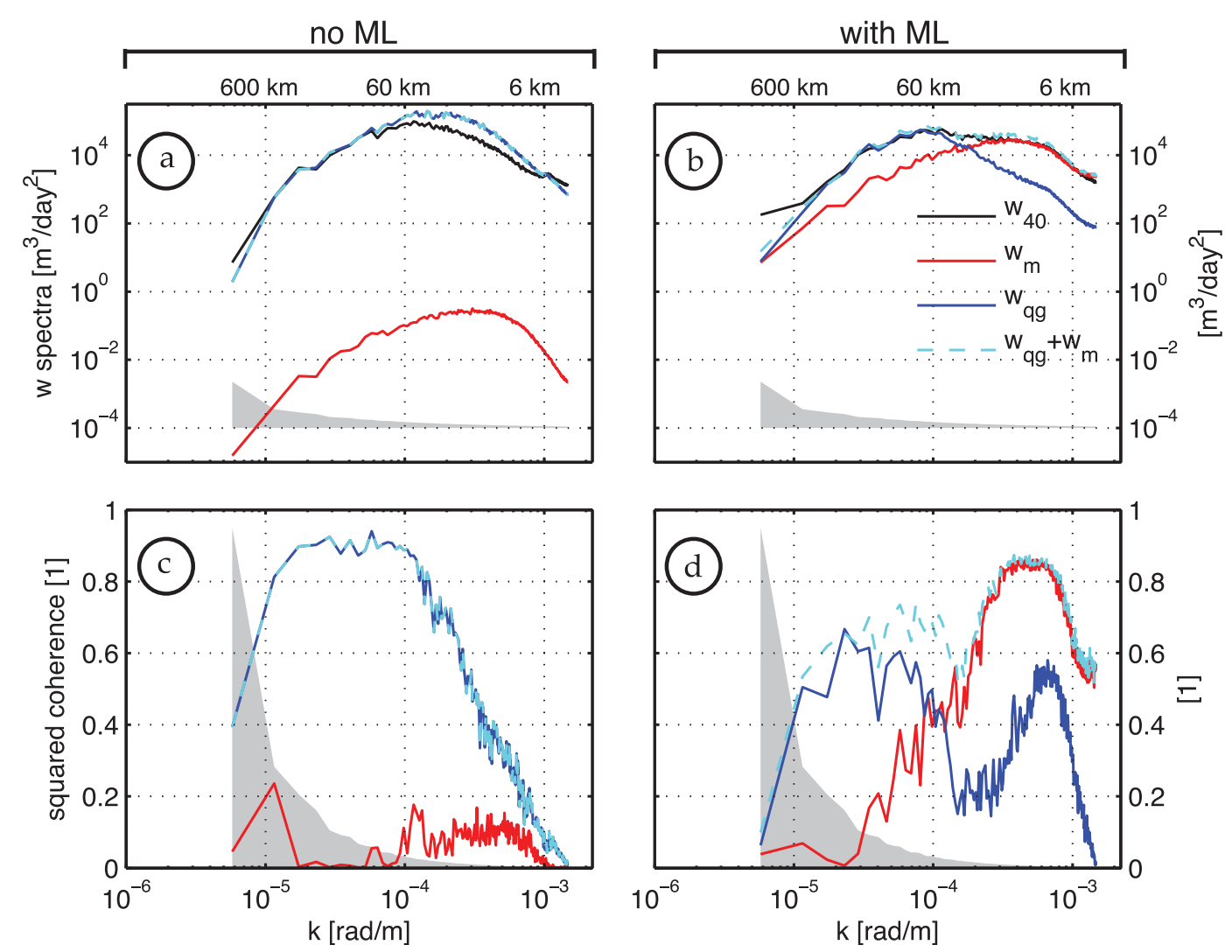

FIG. 4. Spectra of $w_{40}$ (black), $w_{\mathrm{OG}}$ (blue), $w_{m}$ (red), and $w_{\mathrm{OG}}+w_{m}$ (cyan) (a) without and (b) with ML. Units on the vertical axis are in $\mathrm{m}^{3} \mathrm{~s}^{-2}$. On the horizontal axis, $k=10^{-4} \mathrm{rad} \mathrm{m}^{-1}$ corresponds to a wavelength of $60 \mathrm{~km}$. (c),(d) Squared coherence between $w_{40}$ and $w_{\mathrm{OG}}$ (blue), $w_{m}$ (red) and $w_{\mathrm{OG}}+w_{m}$ (cyan) without (c) and with ML in (d). Gray shadings represent $95 \%$ confidence intervals.

the spectrum of $w_{40}$ observed in the simulation (Fig. 4a). The coherence between $w_{40}$ and $w_{\mathrm{QG}}$ is above 0.6- down to $20-\mathrm{km}$ wavelengths (Fig. 4c). Without the ML, vertical motions are therefore well diagnosed using the adiabatic QG framework, which is remarkable since the Rossby number in the surface layers is significant.

A step toward explaining the departures of ML motions from the adiabatic QG diagnosis is made by searching in the momentum equations what terms, other than pressure and Coriolis ones, make the wind-driven ageostrophic motions significant. First, nonlinear advection terms involving only geostrophic motions are unlikely at work since results without an ML indicate a close geostrophic equilibrium over a large spectral range. A detailed analysis of the equations governing the wind-driven ageostrophic motions [see appendix (A1) and (A2)] indicate that these motions are controlled by other nonlinear advection terms involving both geostrophic and ageostrophic motions (these nonlinear terms are usually related to the nonlinear Ekman effects, as noted by Stern 1965; Niiler 1969; Klein and Hua 1988; Thomas and Rhines 2002), and by the QG friction terms, for example, $A_{v} \mathbf{u}_{g z z}$ (Garrett and Loder 1981; Nagai et al. 2006). These studies showed that both nonlinear Ekman and QG friction effects preferentially operate in frontal regions with small length scales.

In the present study surface motions with scales larger than $O(60 \mathrm{~km})$ are not affected by wind-driven ML dynamics. Only smaller-scale motions are affected. The vertical scale of these small-scale horizontal geostrophic motions is actually consistent with the ML depth $H$ [using $N / f \approx 35$, see also (3)]. Then, using $H$ as the depth scale of the geostrophic motions affected by the wind-driven ML dynamics, the resulting Ekman number (related to the ratio between the Ekman depth $\delta_{e}$ and the depth scale of the geostrophic motions affected by the ML dynamics) is $\mathrm{Ek}=\delta_{e}^{2} / H^{2}=0.2$. A scaling analysis (see appendix) indicates that, because of the $O$ (1) nondimensional parameters (Ek, Ro) and the large ratio $u_{\text {Eddy }} / u_{w}$, the QG friction terms should dominate over nonlinear advective terms. A further confirmation is given by the strong correlation between the ML vertical motions and the Laplacian of density at small scales (Fig. 4d), as expected when QG friction terms dominate 
(Garrett and Loder 1981; Nagai et al. 2006), and not with the density gradient or $\zeta$ gradient as when nonlinear Ekman effects dominate (see Stern 1965; Klein and Hua 1988; Thomas and Rhines 2002). QG friction terms are consequently expected to be the leading order terms controlling ageostrophic motions and therefore only vertical mixing terms are retained in the subsequent analysis. The relative success of the analysis is another confirmation that it is an appropriate choice.

\section{Impact of vertical mixing}

A vertical average of the linear momentum equations over the ML depth $H$ (assumed constant over the whole domain) with the boundary conditions $A_{v} \partial_{z} \mathbf{u}(z=0)=\tau / \rho_{o}$ and $A_{v} \partial_{z} \mathbf{u}(z=-H)=0\left(A_{v}\right.$ is the viscosity) leads to

$$
-f \boldsymbol{k} \times \mathbf{u}_{e}=\int_{-H}^{0}-\frac{1}{\rho_{o}} \nabla p(x, y, z) d z / H+\frac{\boldsymbol{\tau}}{\rho_{o} H},
$$

with $\mathbf{u}_{e}$ the estimated total horizontal current (including both geostrophic and ageostrophic components) averaged over the ML, and $\tau$ the wind stress. The pressure gradient $\nabla p$ is assumed to be determined by the mesoscale turbulent eddy dynamics. The ML depth is related to vertical mixing and therefore to wind stress. Thus, (2) is forced by the eddy field through $\nabla p$ and the large-scale wind stress through $\tau$. We assume that pressure can be estimated from the SSH using modified SQG relations which account for the interior PV through an effective Brunt-Väisälä frequency $N_{e}{ }^{1}$ In horizontal spectral space the pressure is given by

$$
\hat{p}(\mathbf{K}, z)=\frac{g}{f} \hat{\eta}(\mathbf{K}) \exp \left(\frac{K N_{e}}{f} z\right),
$$

where the caret stands for the horizontal Fourier transform and $\mathbf{K}=\left(K_{x}, K_{y}\right)$ is the wavenumber vector. After Fourier transforming (2) and using (3), we obtain

\footnotetext{
${ }^{1}$ SQG relations are based on the $\mathrm{PV}$ inversion assuming zero PV in the interior and a nonzero surface PV given by either the SSH or surface density anomalies (Held et al. 1995; Hakim et al. 2002). Modified SQG relations are identical to original SQG relations except for the use of a constant Brunt-Väisälä frequency $N_{e}$ [that may differ from the observed one, $N(z)$ ], which effectively takes into account the contribution from nonzero interior PV [see Lapeyre and Klein (2006), for the derivation]. Usually $N_{e}$ corresponds to $N(z)$ averaged over the first $300 \mathrm{~m}$ (Lapeyre and Klein 2006; Klein et al. 2008, 2009; Lapeyre 2009). These modified SQG relations have been used successfully in several studies (IsernFontanet et al. 2006; Klein et al. 2008; Isern-Fontanet et al. 2008; Klein et al. 2009; Lapeyre 2009).
}

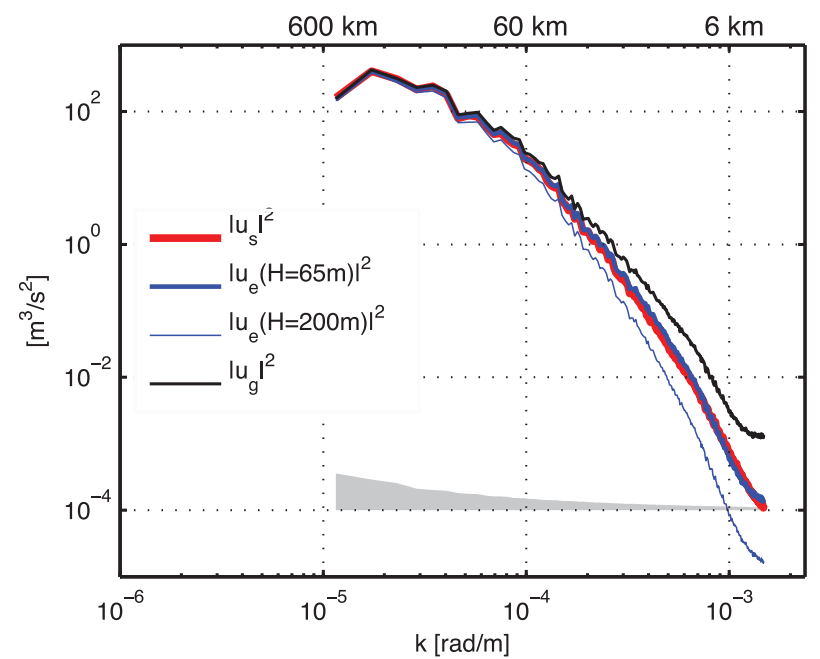

FIG. 5. Surface velocity spectrum estimated from the SSH using geostrophy (black curve), from the velocity observed at the surface (red curve) and from (4) and $\mathbf{u}_{g}$ (using $N_{e} / f=35$ ) (blue curve) in the simulation with a 65-m deep ML. Units on the vertical axis are in $\mathrm{m}^{3} \mathrm{~s}^{-2} ; k=10^{-4} \mathrm{rad} \mathrm{m}^{-1}$ corresponds to a wavelength of $60 \mathrm{~km}$. The thin blue curve corresponds to a surface velocity spectrum estimated from (4) for an ML depth of $200 \mathrm{~m}$. Gray shadings represent $95 \%$ confidence intervals.

$\hat{\mathbf{u}}_{e}\left(K_{x}, K_{y}\right)=\hat{\mathbf{u}}_{g}\left(K_{x}, K_{y}, 0\right) \frac{f}{K N_{e} H}\left[1-\exp \left(\frac{-K N_{e} H}{f}\right)\right]$.

The wind stress does not explicitly appear in (4) since it is large scale and only scales smaller than $O(500 \mathrm{~km})$ are considered here. Vertical mixing indirectly affects horizontal motions through $H$. In (4) its impact is suggested to be stronger for small scales than for large ones as smaller scales (large $K$ ) have smaller vertical extension $\left(f / K N_{e} \lesssim H\right)$ and are more efficiently damped. At last, the coherence between $\mathbf{u}_{e}$ and $\mathbf{u}_{s}$ is close to that between $\mathbf{u}_{g}$ and $\mathbf{u}_{s}$ since only the wavenumber modulus appears in (4).

With the ML, there is a remarkable resemblance between $\left|\hat{\mathbf{u}}_{e}(K)\right|^{2}$ (thick blue curve on Fig. 5), which is estimated from (4) using $\left|\hat{\mathbf{u}}_{g}(K, 0)\right|^{2}$ (black curve on Fig. 5), and the spectrum of the modeled surface velocity $\left|\hat{\mathbf{u}}_{s}(K)\right|^{2}$ (red curve on Fig. 5). This emphasizes the impact of the vertical mixing on horizontal motions with scales smaller than $60 \mathrm{~km}$. The coherence between $\mathbf{u}_{e}$ and $\mathbf{u}_{s}$ is close to one, as mentioned earlier. Thus (4) provides an accurate estimate of $\mathbf{u}_{s}$ based on SSH, the ML depth, and the effective Brunt-Väisälä frequency.

This relative success prompted us to analytically investigate the effects of mixing on vertical motions. For that purpose, we use the simple analytical solution of Garrett and Loder (1981), which is obtained from the 


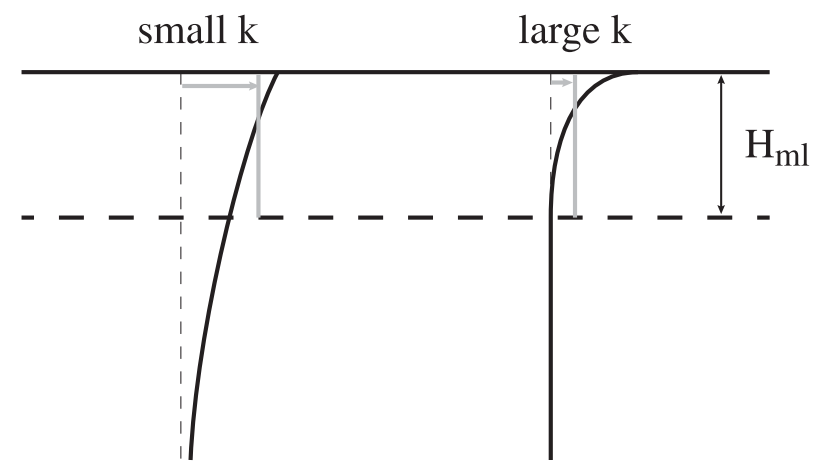

FIG. 6. Illustration of the effect of mixing on the mixed layer QG turbulence and resulting scale selection. Black profiles represent typical SQG profiles, and gray lines and arrows represent the smoothing of these profiles by mixing. For small horizontal length scales (small $k$ ), the effect of mixing is larger.

Omega equation forced by the mixing component of the Q vector (see Giordani and Caniaux 2005; Nagai et al. 2006). This diabatic contribution to the $w$ field is proportional to the Laplacian of density $\nabla^{2} \rho$ (Garrett and Loder 1981):

$$
w_{m} \approx \frac{g}{f^{2} \rho_{o}} A_{v} \nabla^{2} \rho
$$

The $w_{m}$ patterns thereby predicted are fully aligned with and astride surface density fronts (Nagai et al. 2006). The turbulent viscosity $A_{v}$ is here set to a typical ML value of $4 \times 10^{-2} \mathrm{~m}^{2} \mathrm{~s}^{-1}$. In Fig. $4 \mathrm{~b}$, the spectrum of $w_{\mathrm{QG}}+w_{m}$ is quite close to the $w_{40}$ spectrum. The contribution of $w_{m}$ is actually dominant at small scales. The spectral coherence between $w_{\mathrm{QG}}+w_{m}$ and $w_{40}$ (Fig. $4 \mathrm{~d}$ ) is larger than 0.8 for wavelengths down to $8 \mathrm{~km}$. There is only a narrow range of scales from 60 - to $30-\mathrm{km}$ wavelengths where the spectral coherence decreases to 0.6 . It seems therefore possible to significantly improve the QG estimates of the vertical velocity field, in particular at small scales, by including the contribution from vertical mixing.

The concomitant increase of vertical motions and decrease of horizontal motions at small scales is consistent with the presence of intensified mixing at the surface and an attempt of the flow to maintain thermal wind balance against this mixing. In (3), indeed, it is suggested that the strength of geostrophic motions decreases with depth and that the decay rates are larger at smaller horizontal scales. As sketched in Fig. 6, vertical mixing smoothes the vertical profile of the total horizontal motions by producing an ageostrophic component that reduces (enhances) the amplitude of these motions near the surface (at the bottom of the ML). This is consistent, overall, with lower $u_{s}$ variances, more pronounced for smaller scales and quantified by (4). Such viscous effects on horizontal motions tend besides to destroy the thermal wind balance. In response, a vertical circulation develops to decrease horizontal density gradients and restore the thermal wind balance [as expressed by (5)]. This is consistent with the $w$-variance increase observed in the small-scale range.

\section{Discussion}

The present study has analyzed the characteristics of a wind-driven mixed layer embedded in a mesoscale eddy field whose dynamics is mostly captured by the SQG dynamics. Results do indicate a departure of surface currents from geostrophy owing to the intense vertical mixing within the ML. For typical ML depths, horizontal scales below $O(60 \mathrm{~km})$ are affected. According to (4) and Fig. 5, the impact of vertical mixing at these scales should be weaker for shallower MLs and larger for deeper MLs. Then a question related to the motivation of this study is: do the present results suggest practical diagnoses of ML motions from satellite observations? Relation (4), which assumes that the mesoscale eddy field is well captured by the SQG dynamics, provides an estimate of horizontal currents from highresolution SSH provided some knowledge of $N_{e} / f$ and ML depth $H$. Both quantities can be obtained either from climatology (de Boyer Montegut et al. 2004) or from the Argo float database (Holte and Talley 2009). Again, $N_{e}$ usually corresponds to $N(z)$ averaged over the first 300-400 m (Lapeyre and Klein 2006; Klein et al. 2008, 2009; Lapeyre 2009). The adiabatic contribution of the vertical motions (i.e., $w_{\mathrm{QG}}$ ) can be computed from SSH using the SQG methodology (i.e., $w_{\text {SQG }}$ ) as in Klein et al. (2009) [their Eqs. (1)-(4)]. On the other hand, the vertical diabatic contribution (i.e., $w_{m}$ ) can be assessed from (5) using the relation $A_{v} \approx 0.08 \tau / f$ (Kundu and Cohen 2002). Characteristics of the wind stress $\tau$ can be obtained either from climatology or satellite observations (Kudryavtsev et al. 2012). Thus the value of $A_{v} \approx 4$ $\times 10^{-2} \mathrm{~m}^{2} \mathrm{~s}^{-1}$ observed in the present study can be found from this relation using the wind stress value $\tau=5$ $\times 10^{-5} \mathrm{~m}^{2} \mathrm{~s}^{-2}$ that matches the wind characteristics of Fig. 1. Surface density may be inferred from satellite sea surface temperature insofar as salinity does not affect much surface density anomalies. The resulting estimate $w_{m}+w_{\mathrm{SQG}}$ is close to $w_{40}$, both in spectral (Fig. 7) and physical (Fig. 8) space, down to smallest scales. The overall results suggest that ML 3D motions with scales between $O(500 \mathrm{~km})$ and $O(10 \mathrm{~km})$ can be diagnosed from high-resolution SSH and SST. This is encouraging within the context of future high-resolution altimeter missions such as SWOT (Fu and Ferrari 2008). 


\section{w with $M L$}
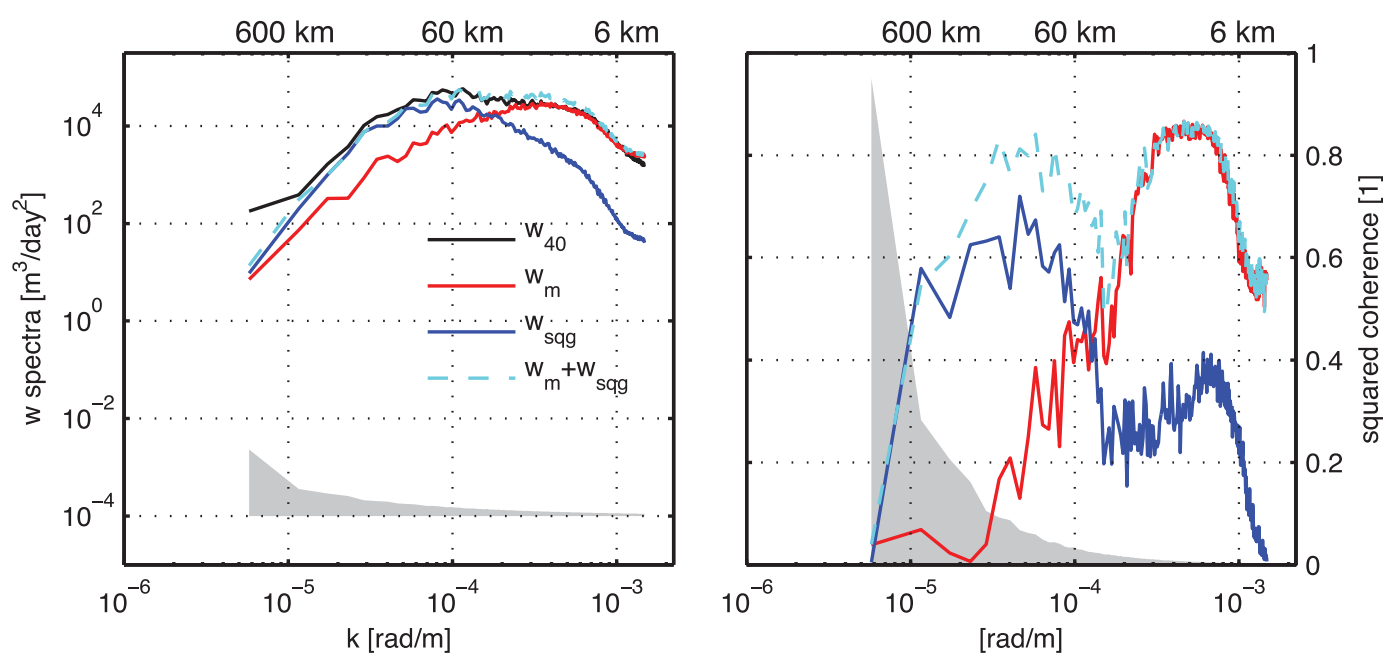

FIG. 7. As in Fig. 3 but $w_{\mathrm{QG}}$ is replaced by $w_{\mathrm{SQG}}$.

The present results are based on several assumptions. First they assume that the mesoscale/submesoscale field is captured by the SQG dynamics. Recent studies indicate that such property is observed in regions with high eddy kinetic energy (Lumpkin and Elipot 2010). SQG turbulent fields are known to involve a large number of fronts/filaments (Held et al. 1995). This property is observed in our numerical simulation where density gradients can exceed $1 \mathrm{~kg} \mathrm{~m}^{-3}(60 \mathrm{~km})^{-1}$ as displayed in Fig. 9a. Although there are not many density gradient observations, some do show such values [see Table 1 in Capet et al. (2008b)]. The present results also assume that the wind-driven velocity scale is much smaller than the eddy velocity scale and that the Ekman number is not too small (which implies a dominance of the vertical mixing as indicated by the scaling analysis of the appendix).

In situations where the wind-driven velocity scale is close to the eddy velocity scale and the Ekman number much less than one (corresponding for example to large ML depths), results of the present study should be no more valid. In such situations the nonlinear Ekman effects may well dominate the viscous effects (Thomas and Rhines 2002). Diagnosis of ML 3D motions in such situations requires an extension of the present study using a much more detailed dynamical approach. Indeed, in situations with large ML depths that favor nonlinear Ekman effects, these effects may not always dominate.
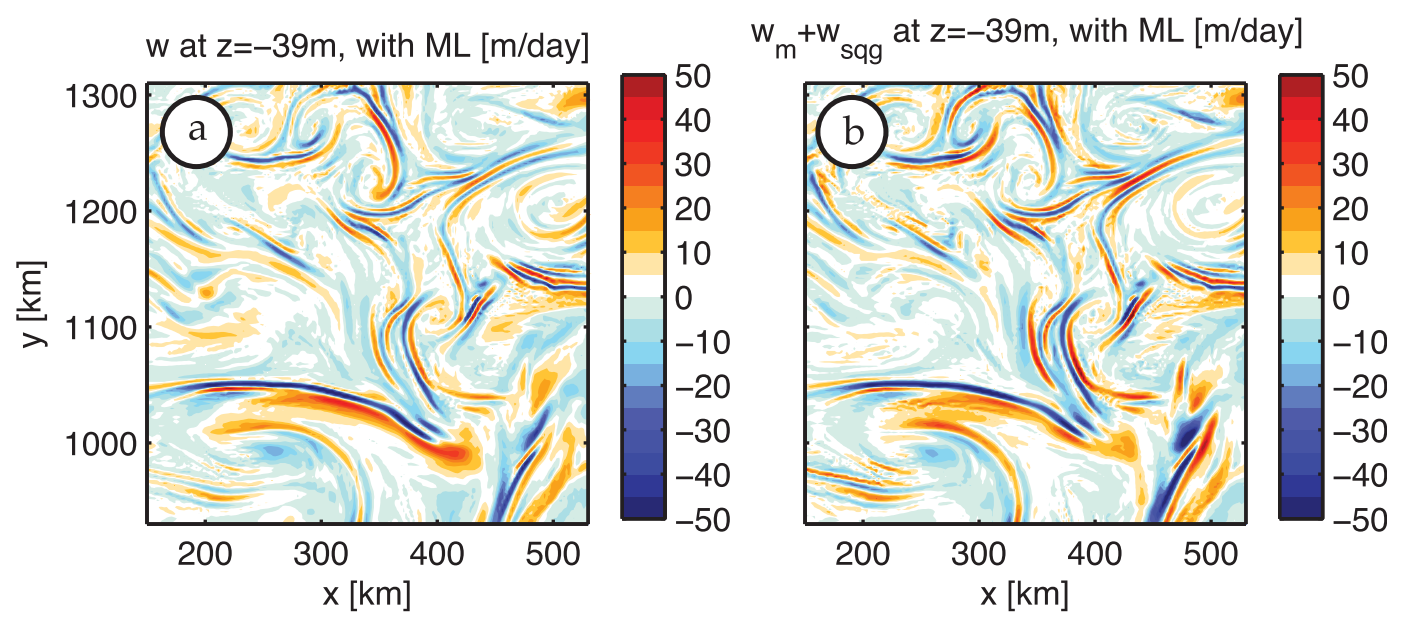

FIG. 8. Snapshots of the vertical velocity field $\left(\mathrm{m} \mathrm{d}^{-1}\right)$ at $40 \mathrm{~m}$ (a) with ML and (b) reconstructed field $w_{m}+w_{\mathrm{SQG}}$. 


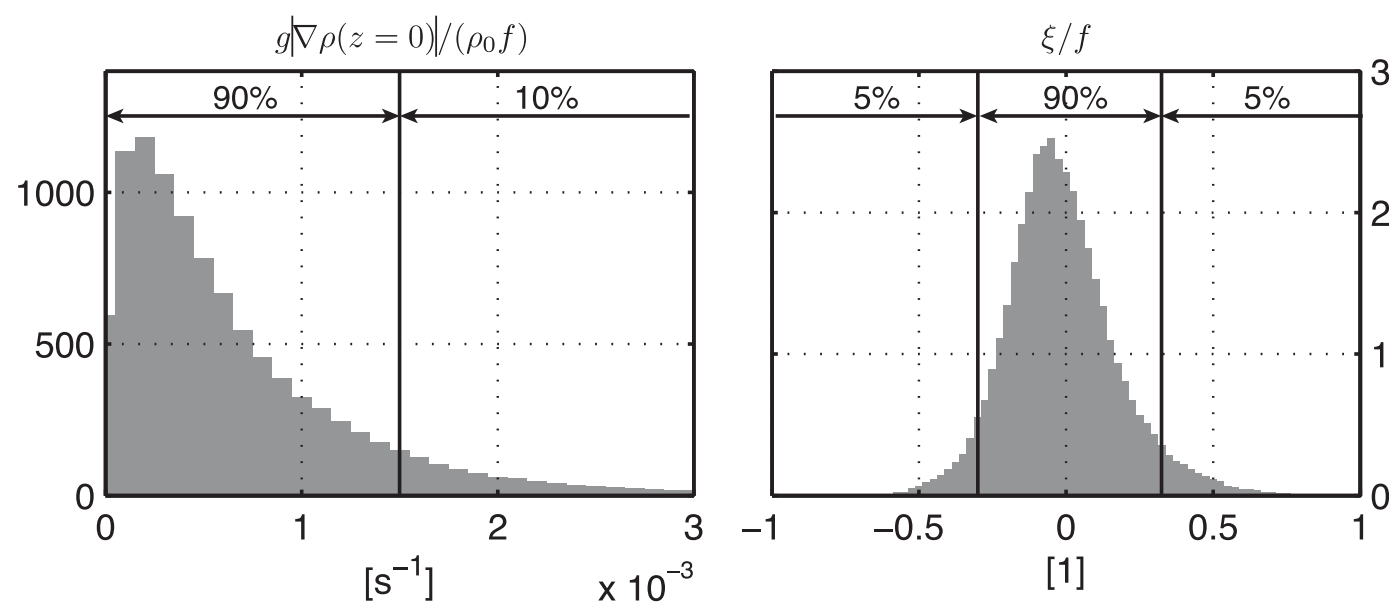

FIG. 9. (a) Probability density function of the surface density gradients $\left[10 \%\right.$ of the gradients are large than $1 \mathrm{~kg} \mathrm{~m}^{-3}$ $(60 \mathrm{~km})^{-1}$ ] and (b) probability distribution function of $\zeta / f$ for the simulation with ML.

Submesocales have been actually found to quickly vary with a time scale close to $f^{-1}$ (in particular when the Rossby number is not small). Furthermore, wind direction also quickly varies at scales of $f^{-1}$ (see Fig. 1). So when considering a time scale longer than $f^{-1}$, the nonlinear Ekman effects may be inhibited by these highfrequency variations. This has to be further examined. On the other hand, with large ML depths, ML frontal dynamics can involve more complex physics (Boccaletti et al. 2007; Thomas and Ferrari 2008; McWilliams et al. 2009; Ferrari 2011; Thomas et al. 2013) such as mixed layer instabilities. This should also make the competition between nonlinear Ekman and viscous effects not straightforward. A last question is related to the wind stress spatial variability. In the present study wind stress is high frequency in time but uniform in space. The potential impact of a nonuniform wind stress has to be contrasted depending on the situation considered. In terms of the vertical velocity induced by the wind stress curl, a rough estimation indicates that a wind stress magnitude $\tau=10^{-4} \mathrm{~m}^{2} \mathrm{~s}^{-2}$ varying over $20 \mathrm{~km}$ should produce a vertical velocity not greater than $4 \mathrm{~m} \mathrm{~d}^{-1}$, a magnitude much smaller than those reported in the present study. So, nonuniform wind stress should have negligible impact in the present study, but in other situations, where the eddy velocity scale is close to the wind-driven velocity, this impact may have to be taken into account. All of these questions should be addressed in a future study.

Acknowledgments. This work is supported by IFREMER, CNRS (FRANCE), the Agence Nationale pour la Recherche [Contracts ANR-05-CIGC-010, ANR-09-BLAN-0365-02 and ANR-10-LABX-19-01 (LabexMER)]. Simulations reported here were done on the
Earth Simulator (Yokohama, Japan) through an M.O.U. signed between IFREMER, CNRS, and JAMSTEC.

\section{APPENDIX}

\section{Nonlinear Ekman Effects versus Viscous Effects (or QG Friction Terms)}

As shown in this scaling analysis and in previous studies (Garrett and Loder 1981; Nagai et al. 2006; Stern 1965; Niiler 1969; Klein and Hua 1988; Thomas and Rhines 2002; Thomas and Ferrari 2008), three nondimensional parameters can be used to determine the relative importance of nonlinear Ekman effects and viscous effects on the ML dynamics: the Rossby number, the ratio $u_{\text {Eddy }} / u_{w}$, and the Ekman number related to the ratio between the Ekman depth and the depth scale of geostrophic motions affected by the ML dynamics (i.e., the ML depth).

We consider a wind-driven mixed layer embedded in a mesoscale eddy field. Motions are decomposed into geostrophic motions $\mathbf{u}_{g}$ and wind-driven ageostrophic motions $\mathbf{u}_{a}$. Assuming geostrophic motions are not affected by ageostrophic motions, the resulting primitive equations for $\boldsymbol{u}_{a}$ are

$$
\begin{aligned}
& u_{a t}+u_{g} u_{a x}+v_{g} u_{a y}+u_{a} u_{g x}-v_{a}\left(f-u_{g y}\right) \\
& =A_{v} u_{g z z}+A_{v} u_{a z z} \text { and } \\
& v_{a t}+u_{g} v_{a x}+v_{g} v_{a y}+v_{a} v_{g y}+u_{a}\left(f+v_{g x}\right) \\
& =A_{v} v_{g z z}+A_{v} v_{a z z} \text { : }
\end{aligned}
$$

$A_{v}$ is assumed constant, and $\mathbf{u}_{a} \nabla \mathbf{u}_{a}$ negligible. Subscripts refer to partial derivatives. Ageostrophic motions are 


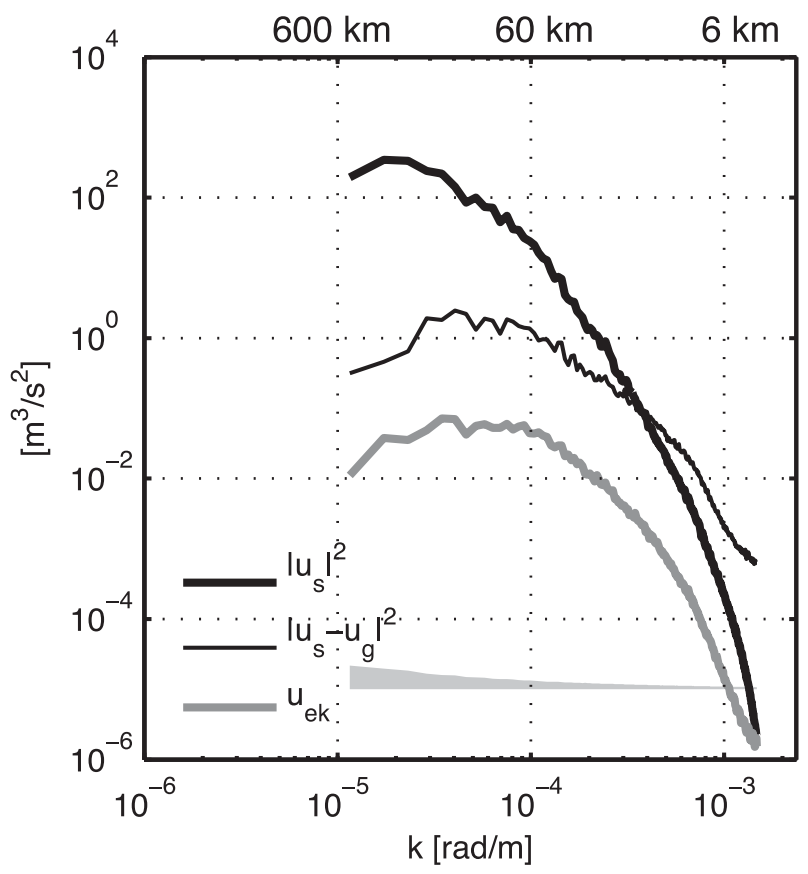

FIG. A1. Spectra of surface current $\left(\mathbf{u}_{s}\right)$, ageostrophic current $\left(\mathbf{u}_{s}-\mathbf{u}_{g}\right)$, and of $u_{\mathrm{Ek}}=u_{w} f /(f+\zeta)$ with $u_{w}=\tau_{\mathrm{rms}} / f H$ in the simulation with ML. Gray shadings represent $5 \%$ confidence intervals.

thus driven by the QG friction terms $\left(A_{v} \mathbf{u}_{g z z}\right)$, or the viscous effects, and the geostrophic current shears present in the nonlinear terms (related to the so-called nonlinear Ekman effects as noted by Stern 1965; Niiler 1969; Klein and Hua 1988; Thomas and Rhines 2002). These terms statistically impact the Ekman transport through the factor $f /(f+\zeta / 2)$ with $\zeta$ the relative vorticity (Klein and Hua 1988). To better assess the impact of large $\zeta$ values, we have examined the probability density function (pdf) of $f /(f+\zeta)$ [instead of $f /(f+\zeta / 2)]$ for the simulation with ML. This pdf is skewed (as the $\zeta$ pdf, see Fig. 9b) but does not exhibit amplitudes much larger than 1: less than $10 \%$ of the total area concern values larger than $1.3 / f$. Then the approximation $\zeta / f \ll 1$ can be considered as valid. Note that the spectrum of $u_{w} f(f+$ $\zeta)$, which can be compared to the spectrum of ageostrophic motions, exhibits much smaller amplitudes (Fig. A1).

To proceed with this scaling analysis we consider the simple case of a meridional geostrophic jet $\left[v_{g}=v_{g}(x, z)\right]$ in thermal wind balance $\left[v_{g z}=-g \rho_{x} /\left(f \rho_{o}\right)\right]$ and a uniform wind stress parallel to the jet $\left(\tau_{y} \neq 0, \tau_{x}=0\right)$. We assume that $\left.A_{v} v_{a z}\right|_{z=0}$ can be approximated as $\tau_{y} / H$ (see Thomas and Ferrari 2008). Then, (A1) and (A2) become

$$
-f v_{a}=A_{v} u_{a z z} ; u_{a}\left(f+v_{g x}\right)=A_{v} v_{g z z}+\tau_{y} / H .
$$

Using $u_{a}=u_{a}^{\prime}+u_{w}$ (with $u_{w}=\tau_{y} / f H$ the mean Ekman velocity), solutions of (A3) are

$$
\begin{aligned}
u_{a}^{\prime}= & \frac{A_{v} v_{g z z}-u_{w} v_{g x}}{f+v_{g x}} \text { and } \\
u_{a x}^{\prime}= & -w_{z}=A_{v}\left[\frac{v_{g z z x}}{f+v_{g x}}-\frac{v_{g z z} v_{g x x}}{\left(f+v_{g x}\right)^{2}}\right] \\
& -u_{w}\left[\frac{v_{g x x}}{f+v_{g x}}-\frac{v_{g z x} v_{g x}}{\left(f+v_{g x}\right)^{2}}\right] .
\end{aligned}
$$

The presence of both $u_{w}$ and the factor $\left(f+v_{g x}\right)$ on the rhs of (A4)-(A5) is related to nonlinear Ekman effects. We can first ask whether viscous terms or $u_{w}$ terms dominate in (A4), which leads to estimate the ratio

$$
R=\left(\frac{A_{v} v_{g z z}}{f}\right) /\left(u_{w} \frac{v_{g x}}{f}\right) .
$$

The term $A_{v} v_{g z z} / f$ corresponds to the ageostrophic motion induced by the QG friction term (Thomas and Ferrari 2008) and $u_{w} v_{g x} / f$ the ageostrophic motion due to the nonlinear Ekman effects. Using $v_{g z z} \approx u_{\text {Eddy }} / H^{2}$ and $v_{g x} / f \approx \mathrm{Ro} / 2$ [valid for a turbulent eddy field (Klein and Hua 1988)] leads to

$$
R \approx 2 \mathrm{EkRo}^{-1} \frac{u_{\text {Eddy }}}{u_{w}} \approx 26,
$$

with $\mathrm{Ek}=0.2$, and $\mathrm{Ro}=0.2 . \mathrm{In}(\mathrm{A} 6)$ it is indicated that, in the present study, viscous terms dominate the $u_{w}$ terms. The factor $f+v_{g x}$ is also present in the $A_{v}$ terms and may impact $w_{z}$. But, this impact should be weak since $v_{g x} \ll f$ (cf. Fig. 9b). As a result, the QG friction term $A_{v} \mathbf{u}_{g z z}$ should be the leading term that controls the ageostrophic motions.

This scaling analysis points out the importance of not only the Rossby number but also of the ratio $u_{\text {Eddy }} / u_{w}$ and the Ekman number. In some other studies these nondimensional parameters are different. Thomas and Rhines (2002) for example used Ro $=0.02$ and $\mathrm{Ek}=$ $10^{-4}$ (see their Table 1 ) and $u_{\text {Eddy }} / u_{w} \approx 1$ (because of their wind stress forcing configuration), leading to a $R \approx$ $10^{-2}$, and therefore concluded to the dominance of the nonlinear Ekman effects. In our study, a further confirmation of the dominance of the viscous effects is provided by comparison of the simulation results with (A5). These results point out that the ML vertical motions are strongly correlated, at small scales, with the Laplacian of density (Figs. 4d and 8b). This characteristic only matches the first term of (A5). If other terms in (A5) 
were dominant, the $w_{40}$ patterns would be correlated with the density gradient or the $\zeta$ gradient (Stern 1965; Niiler 1969; Klein and Hua 1988; Thomas and Rhines 2002) and not with the Laplacian of the density extrema.

\section{REFERENCES}

Boccaletti, G., R. Ferrari, and B. Fox-Kemper, 2007: Mixed layer instabilities and restratification. J. Phys. Oceanogr., 37, 22282250.

Capet, X., J. C. McWilliams, M. Molemaker, and A. Shchepetkin, 2008a: Mesoscale to submesoscale transition in the California Current system. Part I: Flow structure and eddy flux. J. Phys. Oceanogr., 38, 29-43.

$\longrightarrow,-,-$, and $\longrightarrow, 2008 \mathrm{~b}$ : Mesoscale to submesoscale transition in the California Current system. Part II: Dynamical processes and observational tests. J. Phys. Oceanogr., 38, 44-64.

Danioux, E., P. Klein, M. Hecht, N. Komori, G. Roullet, and S. L. Gentil, 2011: Emergence of wind-driven near-inertial waves in the deep ocean triggered by small-scale eddy vorticity structures. J. Phys. Oceanogr., 41, 1297-1307.

de Boyer Montegut, C., G. Madec, A. S. Fischer, A. Lazar, and D. Iudicone, 2004: Mixed layer depth over the global ocean: An examination of profile data and a profile-based climatology. J. Geophys. Res., 109, C12003, doi:10.1029/2004JC002378.

Dohan, K., and N. Maximenko, 2010: Monitoring ocean currents with satellite sensors. Oceanography (Washington D.C.), 23, 94-103, doi:10.5670/oceanog.2010.08.

Ferrari, R., 2011: A frontal challenge for climate models. Science, 332, 316-317.

Fu, L. L., and R. Ferrari, 2008: Observing oceanic submesoscale processes from space. Eos, Trans. Amer. Geophys. Union, 48, 488.

Garrett, C., and J. Loder, 1981: Dynamical aspects of shallow sea fronts. Philos. Trans. Roy. Soc. London, A94, 563-581.

Giordani, L. P., and G. Caniaux, 2005: Advanced insights into sources of vertical velocity in the ocean. Ocean Dyn., 56, 513524, doi:10.1007/s10236-005-0050-1.

Hakim, G. J., C. Snyder, and D. J. Muraki, 2002: A new surface model for cyclone-anticyclone asymmetry. J. Atmos. Sci., 59, $2405-2420$.

Held, I. M., R. T. Pierrehumbert, S. T. Garner, and K. L. Swanson, 1995: Surface quasi-geostrophic dynamics. J. Fluid Mech., 282, $1-20$.

Holte, J., and L. Talley, 2009: A new algorithm for finding mixed layer depths with applications to Argo data and Subantarctic Mode Water formation. J. Atmos. Oceanic Technol., 26, 1920-1939.

Hoskins, B. J., I. Draghici, and H. C. Davies, 1978: A new look at the $\omega$-equation. J. Roy. Meteor. Soc., 104, 31-38.

Isern-Fontanet, J., B. Chapron, G. Lapeyre, and P. Klein, 2006: Potential use of microwave sea surface temperatures for the estimation of ocean currents. Geophys. Res. Lett., 33, L15306 doi:10.1029/2006GL027801.

— G. Lapeyre, P. Klein, B. Chapron, and M. Hecht, 2008: Ocean dynamics reconstruction from surface information. J. Geophys. Res., 113, C09005, doi:10.1029/2007JC004692.
Klein, P., and B. L. Hua, 1988: Mesoscale heterogeneity of the wind-driven mixed layer: Influence of a quasigeostrophic flow. J. Mar. Res., 46, 495-525.

— , G. Lapeyre, and W. G. Large, 2004: Wind ringing of the ocean in presence of mesoscale eddies. Geophys. Res. Lett., 31, L15306, doi:10.1029/2004GL020274.

_ , B. Hua, G. Lapeyre, X. Capet, S. L. Gentil, and H. Sasaki, 2008: Upper ocean turbulence from high 3D resolution simulations. J. Phys. Oceanogr., 38, 1748-1763.

—, J. Isern-Fontanet, G. Lapeyre, G. Roullet, E. Danioux, B. Chapron, S. L. Gentil, and H. Sasaki, 2009: Diagnosis of vertical velocities in the upper ocean from high resolution sea surface height. Geophys. Res. Lett., 36, L12603, doi:10.1029/ 2009GL038359.

Kudryavtsev, V., A. Myasoedov, B. Chapron, J. Johannessen, and F. Collard, 2012: Imaging meso-scale upper ocean dynamics using SAR and optical data. J. Geophys. Res., 117, C04029, doi:10.1029/2011JC007492.

Kundu, P. K., and I. M. Cohen, 2002: Fluid Mechanics. Academic Press, $730 \mathrm{pp}$.

Lapeyre, G., 2009: What vertical mode does the altimeter reflect? On the decomposition in baroclinic modes and on a surfacetrapped mode. J. Phys. Oceanogr., 39, 2857-2874.

- and P. Klein, 2006: Dynamics of the upper oceanic layers in terms of surface quasigeostrophy theory. J. Phys. Oceanogr., 36, 165-176.

Lévy, M., P. Klein, A. Tréguier, D. Iovino, G. Madec, S. Masson, and K. Takahashi, 2010: Modifications of gyre circulation by sub-mesoscale physics. Ocean Modell., 34, 1-15, doi:10.1016/ j.ocemod.2010.04.001.

Lumpkin, R., and S. Elipot, 2010: Surface drifter pair spreading in the North Atlantic. J. Geophys. Res., 115, C12017, doi:10.1029/ 2010JC006338.

McWilliams, J. C., F. Colas, and M. Molemaker, 2009: Cold filamentary intensification and oceanic surface convergence lines. Geophys. Res. Lett., 36, L18602, doi:10.1029/2009GL039402.

Nagai, T., A. Tandon, and D. Rudnick, 2006: Two-dimensional ageostrophic secondary circulation at ocean fronts due to vertical mixing and large-scale deformation. J. Geophys. Res., 111, C09038, doi:10.1029/2005JC002964.

Niiler, P. P., 1969: On the Ekman divergence in an oceanic jet. J. Geophys. Res., 74, 7048-7062.

Pollard, R. T., and L. A. Regier, 1992: Vorticity and vertical circulation at an ocean front. J. Phys. Oceanogr., 22, 609-625.

Rudnick, D. L., 1996: Intensive surveys of the Azores Front. 2. Inferring the geostrophic and vertical velocity fields. J. Geophys. Res., 101 (C7), 16291-16303.

Stern, M. E., 1965: Interaction of a uniform wind stress with a geostrophic vortex. Deep-Sea Res., 12, 355-367.

Thomas, L. N., and P. Rhines, 2002: Nonlinear stratified spin-up. J. Fluid Mech., 473, 211-244.

, and R. Ferrari, 2008: Friction, frontogenesis and the stratification of the surface mixed layer. J. Phys. Oceanogr., 38, 25012518.

, J. R. Taylor, R. Ferrari, and T. M. Joyce, 2013: Symmetric instability in the Gulf Stream. Deep-Sea Res., 91, 96-110. 